\title{
Solar wind interaction with the Earth's magnetosphere: the role of reconnection in the presence of a large scale sheared flow
}

\author{
F. Califano ${ }^{1}$, M. Faganello ${ }^{1}$, F. Pegoraro ${ }^{1}$, and F. Valentini ${ }^{1,2}$ \\ ${ }^{1}$ Physics Dept., University of Pisa, Pisa, Italy \\ ${ }^{2}$ Physics Dept., University of Calabria, Arcavacata di Rende, Italy
}

Received: 20 October 2008 - Revised: 16 December 2008 - Accepted: 19 December 2008 - Published: 16 January 2009

\begin{abstract}
The Earth's magnetosphere and solar wind environment is a laboratory of excellence for the study of the physics of collisionless magnetic reconnection. At low latitude magnetopause, magnetic reconnection develops as a secondary instability due to the stretching of magnetic field lines advected by large scale Kelvin-Helmholtz vortices. In particular, reconnection takes place in the sheared magnetic layer that forms between adjacent vortices during vortex pairing. The process generates magnetic islands with typical size of the order of the ion inertial length, much smaller than the MHD scale of the vortices and much larger than the electron inertial length. The process of reconnection and island formation sets up spontaneously, without any need for special boundary conditions or initial conditions, and independently of the initial in-plane magnetic field topology, whether homogeneous or sheared.
\end{abstract}

\section{Introduction}

In many astrophysical and laboratory systems with $\beta$-values of the order of unity, the large scale plasma dynamics is governed by the interplay between plasma flow and magnetic field. At larger values of $\beta$, the plasma flow becomes the main driver of the system evolution. However, even in this limit, magnetic fields can play a key role in the plasma dynamics. Indeed, the possibility of violating (locally) the "ideal" MHD, low frequency evolution, i.e. the linking condition between flow elements by the magnetic field (the socalled "frozen-in law"), allows the system to access ideally forbidden energetic states. The magnetic energy corresponding to such an energetic jump is then sufficient, in principle, to affect the large scale flow dynamics. The process capa-

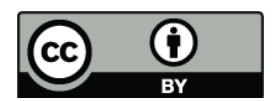

Correspondence to: F. Califano (califano@df.unipi.it) ble of violating the linking condition is known as Magnetic Reconnection (Furth et al., 1963), a fundamental plasma physics process in magnetized plasmas, being the only one capable of affecting, at the same time, the global energy balance of the system, of interest in astrophysics, as well as of reorganizing the large scale topology of the magnetic field, a fundamental point in laboratory fusion plasmas and in theoretical plasma physics.

The theoretical understanding of the physical processes driving the development of magnetic reconnection has progressed strongly over the last few years, mainly by performing an impressive number of large-scale "numerical experiments" of increasing complexity and accuracy. In particular, one of the most important aspects recently investigated is the transition to the so-called "fast reconnection" (Mandt et al., 1994; Shay et al., 1999), in other words the possibility of making reconnection evolving on time scales comparable to the ideal time scale of the large scale system, e.g. the Alfvén time scale. This is needed in order to explain the observed quasi-ideal time scale of energy release, as for example in solar flares, in magnetically confined laboratory plasmas, etc. Possible ingredients, studied by numerical simulation analysis, to make reconnection fast are the development of local "anomalous resistivity" in the MHD regime and the transition to the two fluid regime by the Hall term where electron and ion dynamics separate inside the diffusion layer (see Vekstein and Bian, 2006; Drake et al., 2008, and references therein).

Several fundamental points in the problem of magnetic reconnection still remain to be clarified. For example, even if theoretically the main features distinguishing a resistive or a collisionless reconnection layer are fairly well understood, the debate concerning which micro-process drives reconnection is still open. Another outstanding problem of magnetic reconnection is the interplay between the micro and macrophysics during the full evolution of the large scale system. 
In general, due to the strong analytical and numerical difficulties in solving a inhomogeneous, non-linear, geometrically complex multi-scale problem, magnetic reconnection has been studied in the past mainly by isolating the reconnection process from the full system. Two standard approaches, say an initial value problem or a boundary value problem, have been used. The first one starts from a magnetic configuration, typically a sheared magnetic field with a central neutral line, unstable to reconnection instability. The second approach, instead, makes use of external drivers, as for example the injection of MHD waves, in order to develop reconnection in a "stable" initial magnetic configuration. These studies have shed light on the fundamental aspect characterizing reconnection, i.e. the conditions and the plasma regimes that allow the possibility of demagnetizing the electrons inside very thin "critical" layers, in other words letting the magnetic field lines slip away in such "critical" regions from the electron fluid. In this sense magnetic reconnection can be defined as a "local process". However, not only the consequences of reconnection on the global system, as already discussed, can be important, but also the large scale evolution of the full system creating the conditions for reconnection to occur and the competition between the large-scale system time scale and the development of reconnection time scale are crucial for the "final" configuration of the system. In this sense, the separation between large and small-scale dynamics is somewhat artificial. Magnetic reconnection should not therefore be considered as a "local process", but rather as a multi-scale process. To support this point of view, we cite two important examples. The first one is the Earth Magnetosphere where the structure and dynamics are significantly controlled by magnetic reconnection events involving the Earth and the interplanetary magnetic field. Important consequences are observed on space weather phenomena, e.g. magnetic storms, aurorae. The second well-known example is given by thermonuclear fusion devices where strong internal relaxation oscillations of the central plasma temperature, the so-called saw-tooth oscillations, are driven by the development of reconnection instability. Such relaxations could have important effects on a re-configuration of the equilibrium field with consequences on the plasma confinement efficiency.

The Earth's magnetosphere is one of the best plasma physics laboratories, particularly suited for reconnection studies, where an impressive amount of satellite in-situ measurements of electromagnetic profiles and particle distribution functions are now available. This environment is structured following the interplay between the solar wind and the Earth's magnetic field that results in the stretching of the magnetospheric cavity on the night side and in the transformation of the Earth's magnetic field lines from a dipolar to a large comet-like form. Variations in the interplanetary magnetic field and the solar wind incident on the magnetosphere induce large variations in the magnetospheric system, changing its shape and configuration and altering the number of the flowing currents. Magnetic reconnection has a leading role in many regions of this system. In particular when the interplanetary magnetic field is directed southward, magnetic reconnection with the northward terrestrial field occurs on the dayside magnetospheric boundary; the solar wind then drags the reconnected field lines from the dayside to the night side and further stretches the field lines. As the field lines pile up in the Magnetotail, the system becomes unstable and relaxes through an explosive event called "substorm", which is a violent reconfiguration of the Earth's magnetic tail. The plasma current sheet is disrupted and part of it is redirected toward the ionosphere. Changes in the magnetic topology transform the excess of magnetic energy into fast plasma motions, wave excitations and accelerated charged particles. Plasma motions cover a wide range of scales, from global down to microscopic scales. This dynamics is further complicated by particle bounce motions along closed magnetic field lines of the Magnetotail that occur on nearly the same time scale as reconnection.

Satellite observations by space missions like Geotail, Wind, Cluster etc. have been analyzed over the last few years in order to find reconnection signatures. Geotail measurements were used to give one of the first observation of magnetic reconnection in the Magnetotail (Nagai et al., 2001) with the signature of a Hall current system and a corresponding decoupling of electron and ion dynamics, in agreement with previous hybrid kinetic simulations (Hesse and Winske, 1994). Evidence in satellite data of the collisionless character of reconnection in the Magnetotail was reported also by Oieroset et al. (2001) during a "X-line crossing" inferred by observing a reverse of the Earthward-directed to tailwarddirected plasma jets. During this crossing, the spacecraft recorded a variation of the guide field component with respect to its mean value in agreement with a quadrupolar, out-of-plane configuration of the Hall magnetic field (Hesse and Winske, 1994; Uzdensky et al., 2006; Bian and Vekstein, 2007; Drake et al., 2008). Evidence of Hall effect during reconnection was observed in the dayside magnetopause during a coronal mass ejection event making the current layer thickness comparable to the ion skin depth (Deng and Matsumoto, 2001). In this case, the satellite observed an enhancement of electromagnetic fluctuations in the frequency range on whistler waves in correlation to accelerated plasma flow (considered as a signature of the reconnection process). The authors conclude that their observations agree therefore with the so-called whistler-mediated (fast) reconnection theory (Mandt et al., 1994; Shay et al., 1998). More evidence of Hall physics was observed at the magnetopause where satellite data were used to show that the diffusion region is stable on the ion time scale (Mozer et al., 2002; Vaivads et al., 2004). Recently, coherent magnetic structures (or magnetic islands) with typical dimensions of the order of the ion inertial length and their link to the generation of energetic suprathermal electrons were observed by the Cluster quartet in the Magnetotail (Chen et al., 2008). In situ evidence of magnetic reconnection was even observed in thin current sheets 
forming inside the turbulent magnetosheath, downstream of a collisionless quasi-parallel shock period (Retinó et al., 2007). These current sheets have a typical dimension of a few ion inertial lengths and do not form when the shock is quasiperpendicular. Again, the data seem to be in agreement with a two-fluid, Hall physics picture: 1) inside the so-called "diffusion layer" the electron and ions dynamics separate moving at super/sub-Alfvénic velocity, 2) on the contrary, outside the system behaves as consistent with a MHD description and, finally, 3) the dimensionless estimated reconnection rate turns out to be "fast", $R \simeq V_{i n} / V_{\text {Alfv }} \sim 0.1$. A somewhat different picture of reconnection as a continuous, instead of rather an explosive process can be found during northward solar wind magnetic field periods in the Earth's magnetopause (Frey et al., 2003).

At low latitude magnetopause, the interaction between the Earth's magnetosphere and the solar wind is responsible for the observed denser solar wind plasma entering the Earth side. Here magnetic reconnection could be the driver of the enhanced transport properties when the geomagnetic and the solar wind magnetic field lines are antiparallel, in other words during southward solar wind magnetic field periods. However, a strong mixing is also observed during northward magnetic field periods leading to an increase of the plasma content in the outer magnetosphere even greater than during southward configurations. It has been proposed, therefore, that the shear flow between the solar wind and the magnetosphere drives the formation of Kelvin-Helmholtz (KH) vortices that tend to pair in the non-linear phase (Belmont and Chanteur, 1989; Miura, 1997). This provides an efficient mechanism for the formation of a mixing layer.

Evidence of hydrodynamics vortices has been obtained using the Cluster measurements, apparently with no reconnection signature (Hasegawa et al., 2004). Recently, still based on Cluster satellite data, it has been shown that plasma transport towards the magnetosphere could be caused by magnetic reconnection driven by the development of $\mathrm{KH}$ vortices (Nykyri et al., 2006) and not by the particular orientation of the magnetic field. Indeed, in this region such vortices can develop since the magnetic field is nearly perpendicular to the flow, thus unable to inhibit the KH instability development, leading to large-scale (with respect to the ion inertial length) MHD vortices. However, a small magnetic field component parallel to the solar wind direction is present. This "in-plane" magnetic field, assumed to be sheared in the same plane of the flow, is advected by the rolling-up vortex dynamics and thus increasingly stretched and compressed, eventually leading to secondary reconnection instability on the same scale length of the vortex, the so-called Vortex Induced Reconnection (VIR) (Liu and $\mathrm{Hu}, 1988$ ). The possibility of inducing reconnection by $\mathrm{KH}$ vortices when the in-plane magnetic field is initially parallel to the shear flow, but without any inversion line, has also been demonstrated (Otto and Fairfield, 2000), but on a much smaller time scale than that of the hydrodynamic vortex. Both studies have been made in the context of only one vortex structure, thus preventing the competition with vortex pairing. Furthermore, numerical simulations in conditions of relevance for the low latitude magnetopause, but still limited to a single vortex case, have shown that secondary reconnection instability is able to disrupt the vortex structure, so as to change the large scale evolution significantly (see Nykyri and Otto, 2004, and references therein). However, in the low latitude magnetopause context, another important secondary vortex instability that can develop inside the KH vortices is the Rayleigh Taylor (RT) instability (Smyth, 2003; Matsumoto and Hoshino, 2004). Here the instability is driven by the initial density jump between the solar wind and the magnetosphere leading, after the development of the KH instability, to alternating density layers in the vortex arms. If the initial density difference is strong enough, the vortex centrifugal acceleration, acting as an "efficient gravity", makes the vortex arms RT unstable. The process stops the vortex pairing process and leads finally to the generation of a turbulent mixing layer between the solar wind and the magnetosphere (Faganello et al., 2008b).

In summary, the large-scale system evolution results from a competition between the vortex pairing process (typical of a 2-D hydrodynamic system) and the secondary RT and VIR instabilities. Recently, assuming a homogeneous density distribution in order to focus on reconnection instability only, it has been shown that magnetic reconnection occurs first in the stretched region between two pairing vortices and that, depending on the reconnection time scale development with respect to the system evolution (i.e. vortex rotation time scale), the large scale system can evolve in very different ways ( $\mathrm{Fa}-$ ganello et al., 2008c).

Here we present some recent results obtained using the same model as in Faganello et al. (2008c) but taking an initial in-plane sheared magnetic field.

\section{The model}

In our model, we consider a multi-scale system where the ratio between the box size containing the MHD vortices and the small electron inertial scale is computationally very large, of the order of $L_{x / y} / d_{e} \sim 10^{3}$. In a collisionless plasma, such as that of the low latitude magnetopause, finite ion Larmor radius effects (as well as other kinetic effects like particle trapping, particles acceleration, deformation of the distribution function and so on) would probably play a role even before reaching the electron inertial scale length, thus requiring a full kinetic approach but demanding an enormous computational effort. However, as a first step, we limit our investigation to a large-scale fluid approach, just including electron inertia terms, a key ingredient for collisionless reconnection (Coppi, 1964). Aware of the limits of this approach since kinetic effects could have important feedback on the large scale system evolution, we think that our model can 


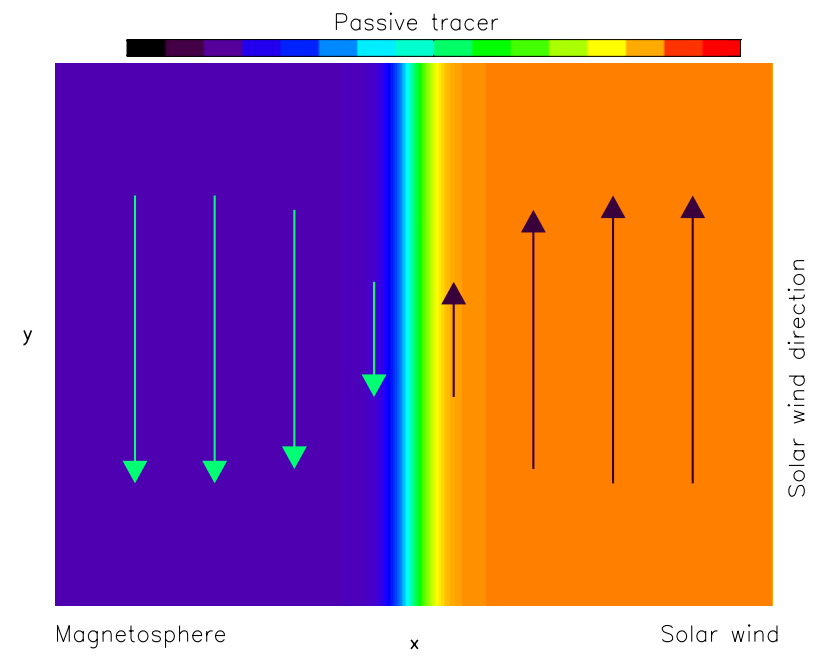

Fig. 1. The initial magnetospheric and the solar wind plasma configuration represented by blue and yellow (dark and pale) passive tracers. The colour intensity is proportional to the velocity flow intensity. Yellow and blue color correspond to positive and negative values of the flow. The arrows represent the direction of the flow as well as of the in-plane component of the magnetic field.

nevertheless give important qualitative information towards the understanding of low latitude magnetospheric physics, as well as important answer concerning the theory of collisionless reconnection driven by the presence of a shear flow. On the same line of our approach, we do not consider of relevance the use of a relatively complicated equation of state, thus using an isothermal closure.

We adopt a two-fluid description of the plasma going from the low frequency MHD regime to the relatively high frequency electron-MHD regime (Attico et al., 2008a) through the ion cyclotron frequency. The plasma is assumed to be quasi-neutral, $n_{i} \simeq n_{e} \equiv n$. All equations are made dimensionless using ion characteristic quantities: the ion mass $m_{i}$, the ion cyclotron frequency $\Omega_{c i}$, the Alfvén velocity $v_{a}$ and therefore the ion skin depth $d_{i}=c / \omega_{p i}$; as a result, the dimensionless electron skin depth corresponds to the mass ratio square root, $d_{e}=\left(m_{e} / m_{i}\right)^{1 / 2}$. We define $\mathbf{U}=\left(\mathbf{u}_{\mathbf{i}}+d_{e}^{2} \mathbf{u}_{\mathbf{e}}\right)$ as the fluid velocity. Furthermore, in this relatively low frequency regime we neglect the displacement current in the Ampere equation, giving a simple relationship between the current, defined as $\mathbf{j}=n \mathbf{u}_{\mathbf{i}}-n \mathbf{u}_{\mathbf{e}}$ (where $n$ is the density) and the magnetic field:

$\mathbf{j}=\nabla \times \mathbf{B}$.

In a quasi-neutral plasma the continuity equation reads:

$$
\frac{\partial n}{\partial t}+\nabla \cdot(n \mathbf{U})=0
$$

Then, by summing the first moment of the electron and proton equation, multiplied by $m_{e}$ and $m_{i}$, respectively, we ob- tain an equivalent equation for the velocity field $\mathbf{U}$ in conservative form:

$\frac{\partial(n \mathbf{U})}{\partial t}+\frac{1}{1+d_{e}^{2}} \nabla \cdot\left[n \overline{\overline{\mathbf{V}}}+\overline{\overline{\mathbf{P}}}_{T}-\mathbf{B B}\right]=0$

where $\overline{\overline{\mathbf{V}}}=\mathbf{u}_{\mathbf{i}} \mathbf{u}_{\mathbf{i}}+d_{e}^{2} \mathbf{u}_{\mathbf{e}} \mathbf{u}_{\mathbf{e}}, \overline{\overline{\mathbf{P}}}_{T}=P_{T} \overline{\overline{\mathbf{I}}}$ and $P_{T}=P_{i}+P_{e}+B^{2} / 2$ is the total pressure. As already discussed, for the sake of simplicity we consider an isothermal closure. Therefore, the fluid pressures in Eq. (3) are given by $P_{i, e}=n T_{i, e}$ using the density calculated from Eq. (2). By subtracting the electron and proton motion equations, making a Boussinesqlike approximation $\left(1 / n \simeq 1\right.$ when multiplied $\left.\nabla^{2} \mathbf{E}\right)$ and taking $\nabla \cdot \mathbf{E} \simeq 0$, we obtain the following generalized Ohm's law (Valentini et al., 2007):

$$
\begin{aligned}
(1+ & \left.d_{e}^{2} \nabla^{2}\right) \mathbf{E}=-\mathbf{u}_{\mathbf{i}} \times \mathbf{B}+-\frac{1}{n}(\mathbf{j} \times \mathbf{B}) \\
- & d_{e}^{2}\left\{\mathbf{u}_{\mathbf{i}} \times \mathbf{B}+\frac{1}{n} \nabla \cdot\left[n\left(\mathbf{u}_{\mathbf{i}} \mathbf{u}_{\mathbf{i}}-\mathbf{u}_{\mathbf{e}} \mathbf{u}_{\mathbf{e}}\right)\right]\right\}
\end{aligned}
$$

that, combined with the Faraday equation

$$
\frac{\partial \mathbf{B}}{\partial t}=-\nabla \times \mathbf{E}
$$

describes the evolution of the magnetic field B. The term $\nabla P_{i, e} / n$ in the generalized Ohm's law has been omitted since, for a polytropic equation of state, it does not contribute to $\nabla \times \mathbf{E}$.

Equations (1-5) are integrated numerically using an explicit, third order accurate, time advancing scheme (AdamsBashfort III) in the spatial domain $L_{x} \times L_{y}$ with open and periodic boundary conditions, respectively. Standard compact finite differences (VI order accurate) and spectral methods are used to calculate the derivatives along the $\mathrm{x}$ - and $y$-directions. In particular, concerning the inhomogeneous $\mathrm{x}$-direction, we implemented transparent boundary conditions in order to let sonic and alfvénic perturbations, at first generated by the compressional $\mathrm{KH}$ instability and then by the strongly nonlinear dynamics, leave the numerical domain. These transparent conditions are obtained from the ideal MHD set of hyperbolic equations for which it is possible to define projected characteristic along the $\mathrm{x}$-direction (see Landi et al., 2005, and references therein), not possible instead for the two-fluid set of equations. The idea is that $\mathrm{KH}$ vortices can be considered as large-scale MHD structures even when the Hall term and electron inertia are included and that, except for the central region where the dynamics separates the electrons and ion dynamics, the MHD approximation holds. Concerning the results presented in this paper, we impose in the numerical code that there are no MHD waves (Alfén, slow and fast) entering in the domain from the left and right boundaries along the full duration of the simulation. On the other hand, as already stated, MHD perturbations are free to leave the domain. Finally, in the code, at each time step the electron and ion fluid velocities are obtained by combining the definitions of the velocity field $\mathbf{U}$ and of the current density $\mathbf{j}$. 


\section{Results}

We model the solar wind interaction with the magnetosphere, at low latitude, by taking at $t=0$ a large-scale, sheared velocity field directed along the y-axis,

$\mathbf{U}_{e q}=\left(U_{0} / 2\right) \tanh \left[x / L_{u}\right] \mathbf{e}_{y}$

The initial mean velocity is carried by both electrons and ion corresponding to a zero total current. Furthermore, we consider a homogeneous initial density, $n=1$ in dimensionless units, thus eliminating the possible development of the secondary RT instability. Given the adopted isothermal closure, we also get that the initial electron and ion pressure are constant. Admittedly, this choice reduces the degree of realism of the model, but allows us to focus our investigation on the physics driven by the development of the secondary reconnection instability. The equilibrium magnetic field is composed by a relatively moderate, sheared in-plane component $B_{y, e q}$ parallel to the initial flow, and by a guide field component $B_{z, e q}$ perpendicular to the reconnection $(x, y)$ plane:

$\mathbf{B}_{e q}(x)=B_{y, e q}(x) \mathbf{e}_{y}+B_{z, e q}(x) \mathbf{e}_{z}$

$B_{y, e q}=B_{0} \tanh \left[x / L_{B}\right]$

$B_{z, e q}=\left[B_{0 z}^{2}+B_{0}^{2}-B_{y, e q}^{2}\right]^{1 / 2}$

where $B_{z, e q}$ is chosen such that the total pressure $P_{T}$ is constant. Note that when the value of $B_{0}$ is "sufficiently" small, $\sqrt{B_{0}^{2} / n} \ll U_{0}$, the KH instability can freely develop without being inhibited by magnetic field line tension. The Eqs. (1-5) are integrated in a 2-D slab geometry (with 3 -D vector fields) in the domain $x \in\left[-L_{x} / 2, L_{x} / 2\right], y \in\left[0, L_{y}\right]$.

\subsection{Vortex pairing and onset of fast reconnection}

In Fig. 1 we show, very schematically, the initial model configuration of the system. The arrows represent the direction of the flow as well as of the in-plane component of the magnetic field. Note that the color levels do not represent a density variation, but just distinguish the solar wind plasma from the magnetospheric plasma. In the following, we present recent results obtained with $m_{i} / m_{e}=64$ and:

$$
B_{0 z}=1 ; \quad B_{0}=0.05 ; \quad T_{e}=T_{i}=0.5 ; \quad L_{u}=L_{B}=3 .
$$

In our dimensionless units, the magnetic guide field at both the left and right boundary is equal to one, $B_{z, e q}( \pm \infty)=1$. The box dimensions $L_{x}=90, L_{y}=30 \pi$ is set to contain twice the fast growing mode wave-length, leading therefore to the development of two KH vortices. This allows us to study the competition between pairing process and vortex disruption caused by the development of the secondary reconnection instability. This choice is the same as in previous studies (Faganello et al., 2008b,c) where, however, the in-plane magnetic field was homogeneous, i.e. $B_{y, e q}=c s t$. In Fig. 2

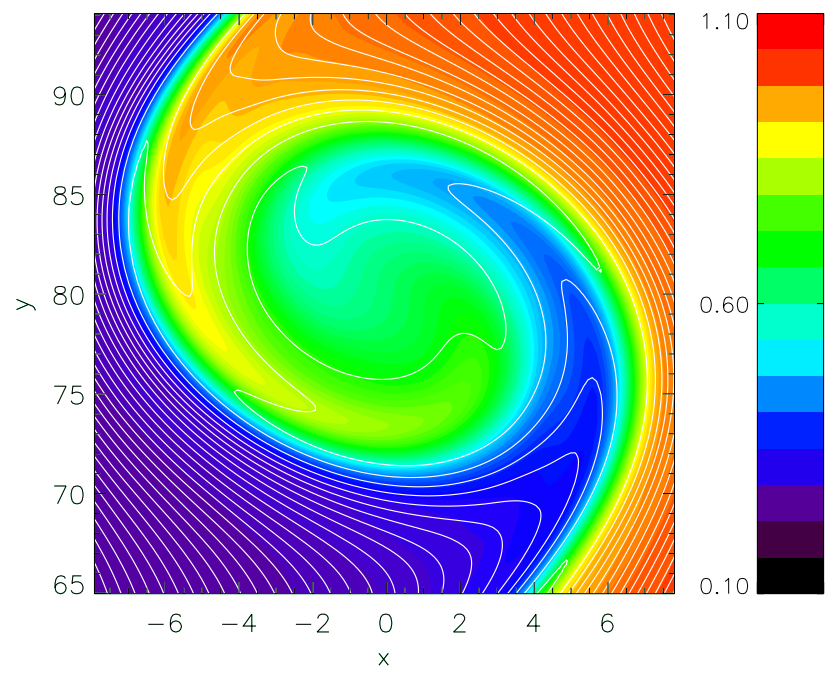

Fig. 2. The plasma passive tracer as defined in Fig. 1 and the inplane magnetic field lines at $t=300$.

we show one of the two vortices generated by the $\mathrm{KH}$ instability at $t=300$. The white line represent the in-plane magnetic field lines. We see that the magnetic lines have already reconnected inside the vortex, close to the neutral line, forming a large scale magnetic island of the order of the vortex size, a clear example of the VIR process (Liu and Hu, 1988). We note that the shape of the island is quite distorted by the rotation motion. Furthermore, more or less at the same time, at the external borders of the vortices, we see several magnetic field lines crossing the neutral line and thus connecting the solar wind plasma to the magnetospheric plasma. The VIR process is driven by the field line stretching induced by plasma motions. The formation of the magnetic islands and the magnetic link established between the solar wind and magnetosphere regions has occurred only because of the initial magnetic shear allowing the connection of initially left lines $(x<0, t=0)$ with initially right lines $(x>0, t=0)$. Such link cannot be established for an initially homogeneous inplane magnetic field where reconnection generates magnetic islands, but on a much smaller scale length and at a much later time with field lines that only belong to the same plasma (solar wind or magnetospheric). The important consequence, investigated for a single vortex case, is that in the homogeneous limit reconnection is unable to significantly enhance the transport since it is only capable of self-reconnecting a field line. Instead, in the presence of an initial sheared in-plane magnetic field, the two side plasmas mix across the neutral line since initially different field lines reconnect (Nakamura and Fujimoto, 2006). In Fig. 3, left frame, we show at $t=460$, in the center region of the simulation box, the two vortices generated by the KH instability that already started to pair. The solar and magnetospheric plasmas alternate inside the vortex arms separated by the magnetic neutral 

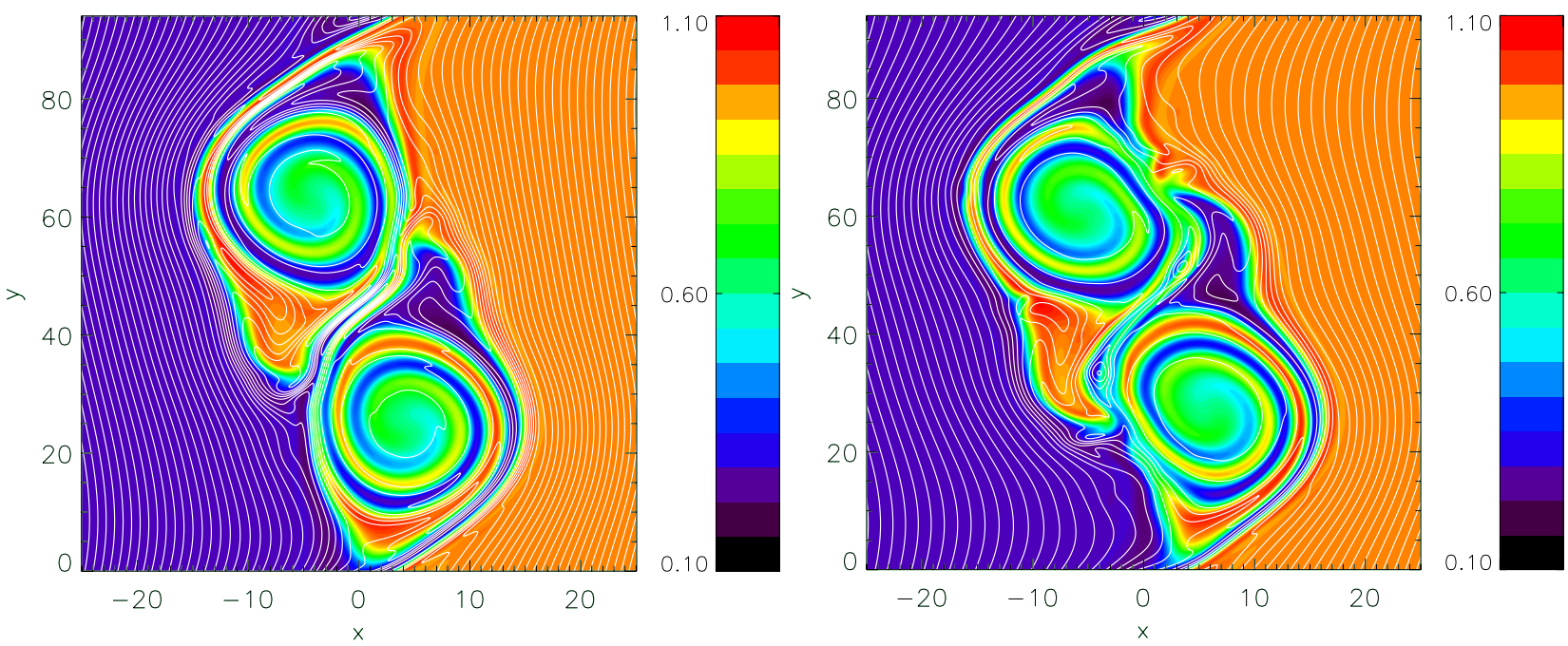

Fig. 3. The plasma passive tracer as defined in Fig. 1 and the in-plane magnetic field lines at $t=460$ and $t=480$, left and right frame.

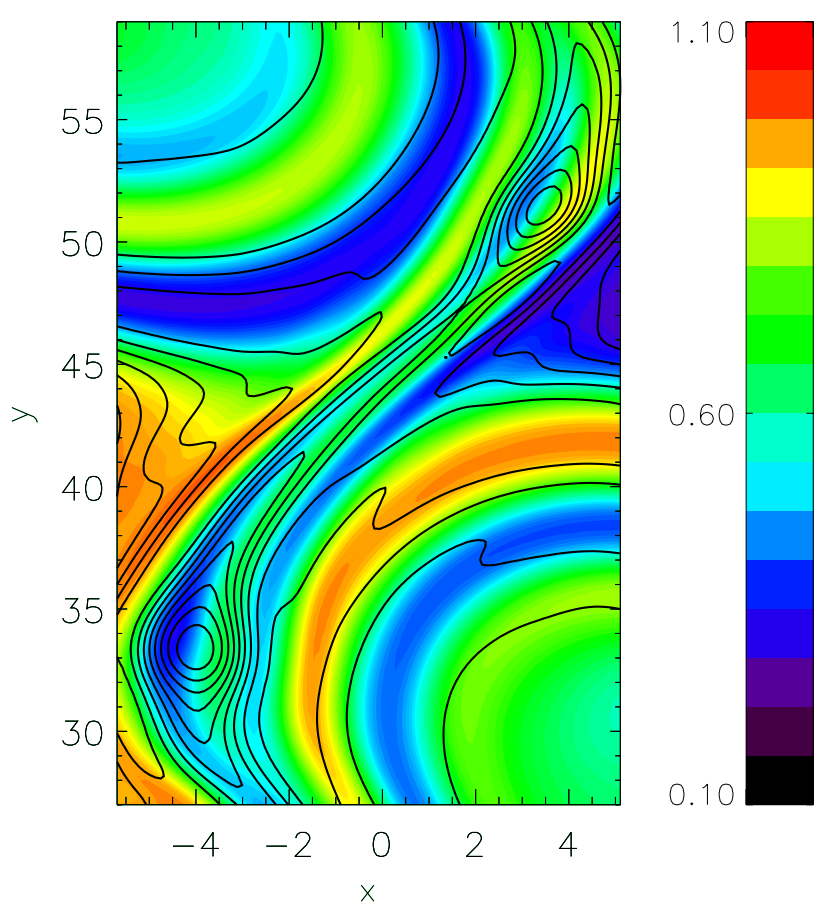

Fig. 4. Zoom of Fig. 3, right frame. For the sake of clearness, magnetic field lines are drown in black.

line as a result of the rolling-up of the $\mathrm{KH}$ vortices. We now see, as a consequence of the progress of the VIR process, many field lines, for example around the point $x=-3$, $y=80$ for the upper vortex, coming from the solar wind side, crossing the neutral line, going through the magnetospheric plasma and coming back into the solar wind plasma (the

same is observed for the lower vortex). But the new important process with respect to VIR is that we now observe the onset of the reconnection instability in the region between the two vortices analogous to the case of a homogeneous initial magnetic field (Faganello et al., 2008b). Indeed, we see the formation of, at least, two X-points, the first one at $x \simeq 4, y \simeq 55$ and the second one at $x \simeq-3, y \simeq 34$. These $X-$ points are both located on the ribbon that connects the two vortices and that is subject to compression due to the pairing process. In the further evolution, magnetic reconnection develops on a "fast" time scale $\tau \sim 10$, where "fast" here means with respect to the system rotation time scale. As a result, the system generates two magnetic islands of size of the order of the ion inertial length visible at $t=480$ in the right frame of Fig. 3, nearby the location of the X points. A clear picture of the two magnetic islands is given in Fig. 4 by a zoom of Fig. 3 (right frame) centered on the X-point located at $x \sim 1, y \sim 45$. For the sake of clearness, magnetic field lines are drown in black. Note that the $\mathrm{X}$ point has moved slightly with respect to its previous position at $t=460$ (see left frame of Fig. 3). In Fig. 5 we plot the $x$ component of the electron and ion velocity vs. $x$ at $t=480$. The figure demonstrate that in the reconnection region the electron and ion dynamics separate corresponding to a transition to a two-fluid regime, while outside the system behaves essentially following single fluid MHD dynamics. The electron flow varies on the electron inertial length, while ion on the smoother ion inertial length.

\subsection{The guide field and the Hall quadrupole}

In Fig. 6 we show, at $t=480$ (same time as in Fig. 3, right frame), the shaded iso-contours of the guide field component of the magnetic field $B_{z}$. The black lines represent 


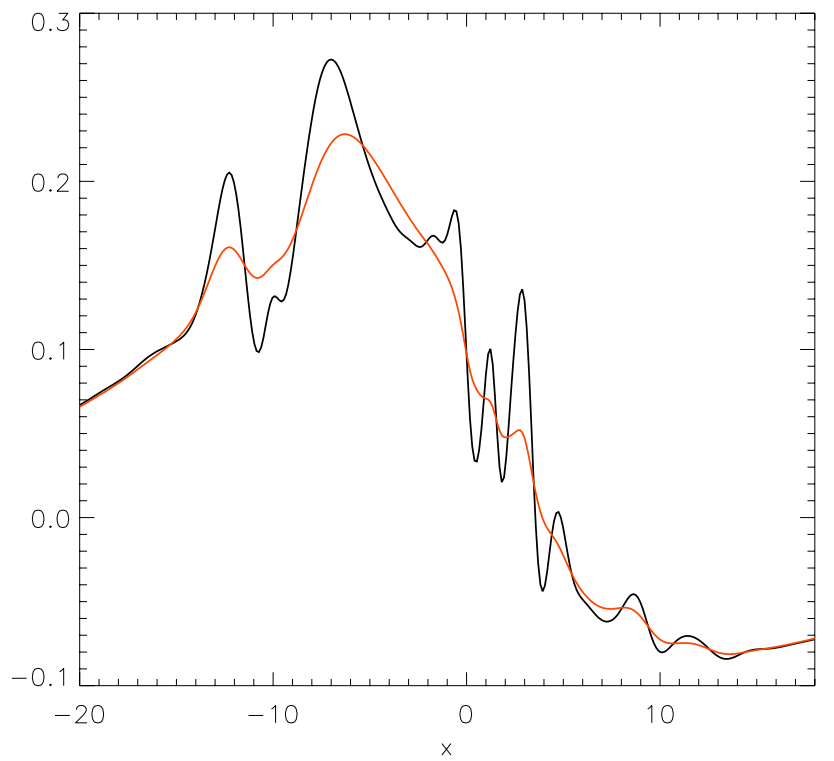

Fig. 5. The ion (red) and electron (black) velocities at $t=480$ vs. $\mathrm{x}$ at $y=47$.

the in-plane magnetic field lines as in Fig. 3. We see that $B_{z}$ is strongly depressed in two regions (blue in the figure) located at the center of the $\mathrm{KH}$ vortices, while it rapidly fluctuates outside around its mean value. More important, crossing the $\mathrm{X}$-point at $(x \approx 1, y \approx 45)$, a signature of the Hall quadrupole is clearly visible around the two small magnetic islands located, the first one, at $x \approx 3, y \approx 50$, the second one at $x \approx-2, y \approx 35$. A zoom of the quadrupole embedded between the two magnetic islands crossing the X-point is given in Fig. 7. It is worth underlining that, instead, in the case of an initial homogeneous magnetic field (Faganello et al., $2008 \mathrm{~b}, \mathrm{c}$ ) we did not observe any significant signature of the Hall term quadrupole, in our opinion caused by the strong plasma compression induced by the plasma flow (Bian and Vekstein, 2007). Here instead, the compressional effects are quantitatively less important, of the order of $10 \%$ at maximum. If we now imagine crossing with a satellite the central region around the X-point in Fig. 6, we would observe many fluctuations of the guide field profile. Most of these variations result from compressive effects induced by plasma motions that produce a filamentary structure of $B_{z}$, while only a few are generated by Hall reconnection. Indeed, by looking at a number of cut plot along the $\mathrm{x}$-axis (not shown here), crossing or not the Hall quadrupole, we obtain very similar profiles of $B_{z}$. As discussed in the introduction, the Hall signature recognized by satellite observations as a fluctuation of the guide field component around its mean value, is considered more or less as evidence of "fast" reconnection. In our opinion, numerical simulations show, at least in the two-fluid approximation, that it is very difficult to distinguish compressive driven fluctuations of $B_{z}$ from Hall quadrupole

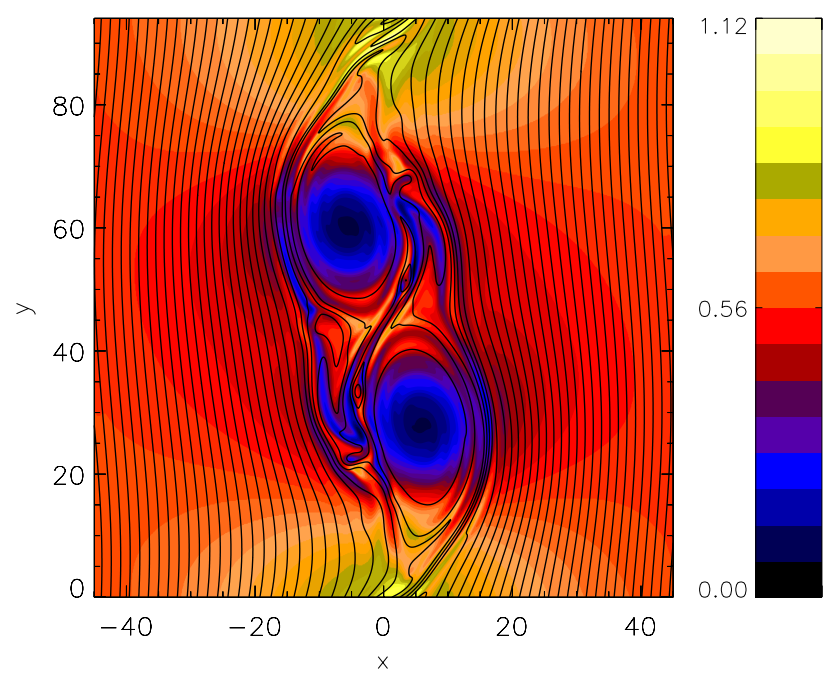

Fig. 6. Shaded iso-contours of the guide field component $B_{z}$ and the magnetic field lines at $t=480$.

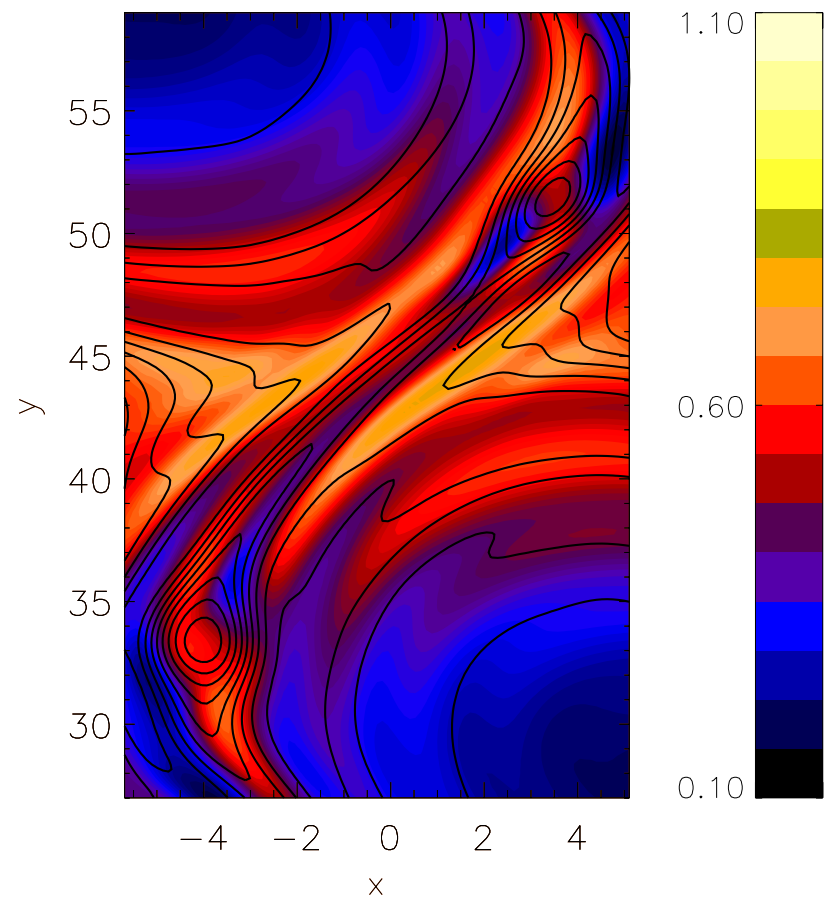

Fig. 7. Zoom of the central region of Fig. 6 .

variations. One should therefore pay attention when interpreting the variation of the guide field component necessary as a signature of Hall reconnection.

\subsection{Compressive effects}

The relevance of field line compression by plasma flow in the system is outlined by the time evolution of the in-plane 

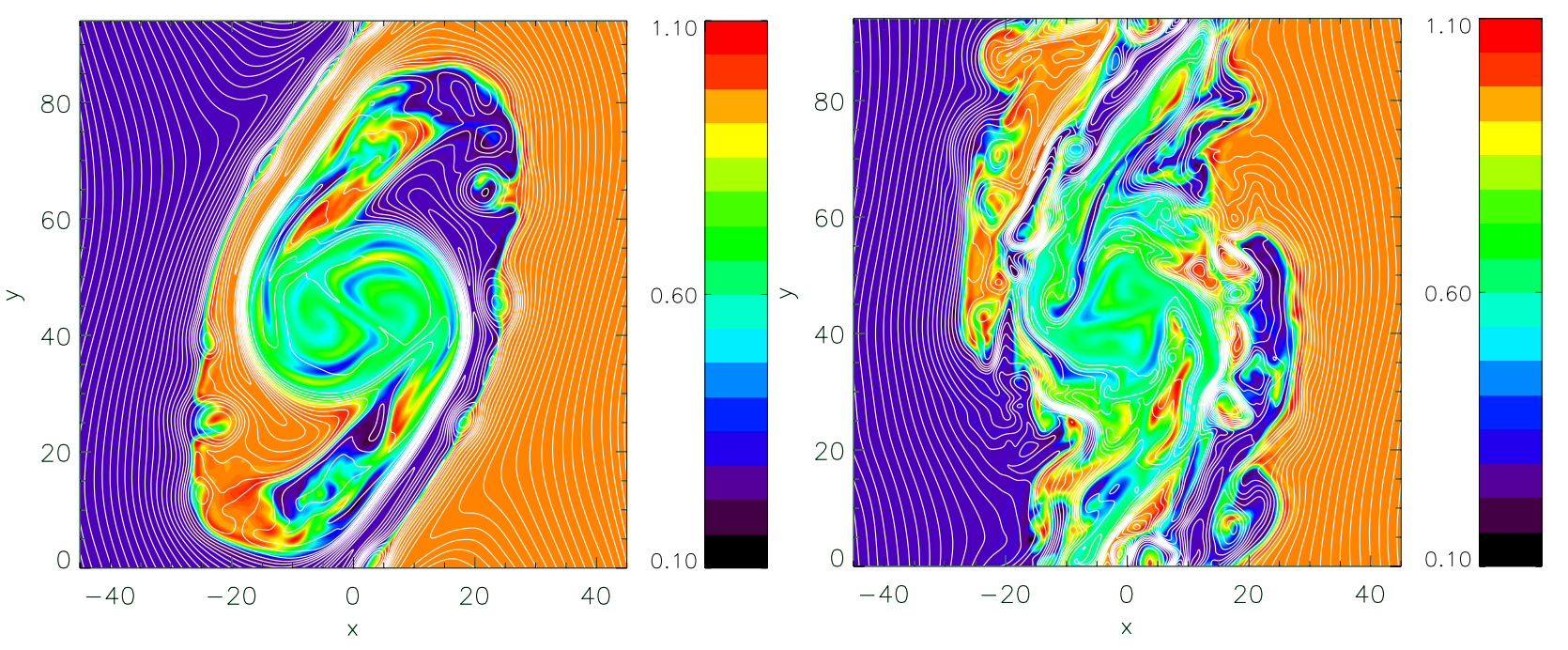

Fig. 8. The plasma passive tracer as defined in Fig. 1 and the magnetic field lines at $t=675$ and $t=825$.

kinetic and magnetic energy, $E_{k, \perp}$ and $E_{m, \perp}$ (not shown here), where the perpendicular direction mean with respect to the guide field direction. First of all, the in-plane magnetic energy, which is initially negligible with respect to the in-plane kinetic energy, $E_{m, \perp}(t=0) \ll E_{k, \perp}(t=0) \equiv E_{k, \perp}^{0}$, increases up to a few percent of $E_{k, \perp}^{0}$ between $300<t<400$, then remains more or less constant until $t=550$ and finally rapidly increases up to almost the $20 \%$ of $E_{k, \perp}^{0}$. Such a strong amplification is however a direct consequence of the field line compression by the flow and not of a dynamo like action of the system since the integral of the magnetic flux (not shown here) is conserved in time (with small fluctuations in the final part of the evolution due to unavoidable small scale grid effects). Nevertheless there is a conversion of kinetic energy, that decreases more than the $20 \%$ of its initial value, into magnetic energy, even if reconnection acts in the opposite way by decreasing the amount of magnetic energy. This is due to the fact that the energetic contribution of the reconnection events is small compared to the effect of magnetic field compression driven by the flow. A similar behavior of the energy and integrated magnetic flux in time is observed in the MHD limit made by running exactly the same simulation, but with the Hall and electron inertia terms replaced by a resistivity term $\eta \mathbf{j}\left(\eta=10^{-3}\right)$ in the generalized Ohm law, Eq. (5).

\subsection{Long time evolution}

In Fig. 8 we show the plasma tracers and the magnetic field lines at later times, $t=675$ and $t=825$, left and right frame. Aware that pushing a two-fluid simulation so far is unrealistic, we are nevertheless convinced that these simulations can produce qualitative large scale results to be considered in this context as a first, but important step. In the left frame of Fig. 8 we see that the pairing process finally generates a single vortex structure. We therefore conclude that in this system the pairing process is more rapid and efficient in generating a single vortex structure than the secondary reconnection instability in disrupting each initial single vortex; this is a different result from that found in the case of the secondary RT instability (but with no in-plane magnetic field), acting in the presence of a sufficiently strong density jump, that was able to disrupt each vortex so rapidly to inhibit the pairing process (Faganello et al., 2008a). Due to the stretching induced by the plasma motions during the rotation and merging of the initial $\mathrm{KH}$ vortices, the two magnetic, relatively small islands visible at $t=480$ (Fig. 3 right frame) have now been destroyed. In the mean time, however, several new magnetic islands, still of size comparable to the ion scale length, have been generated mainly at the vortex boundary between the solar wind and the magnetospheric plasma (see Fig. 8, left frame). These structures are now quite stable and are advected by the system conserving more or less their shape and typical size, at least until the end of the simulation, $t=825$, as shown by the right frame of Fig. 8. Again, our model was pushed too far to be compared directly with satellite observations, but we conjecture that the mechanism of generation of such magnetic islands on the ion inertial scale length could be considered as a good candidate in order to account for the magnetic structures observed by the satellites on the ion scale length (Hasegawa et al., 2005).

A very important consequence of the generation of the magnetic islands at the boundary between the two plasmas is the possibility of connecting the solar wind plasma with the magnetospheric plasma, thus in principle strongly enhancing the mixing efficiency. For example, at $t=675$, left frame of Fig. 8, the upper (lower) magnetospheric field lines, say $y=L_{y},-25<x<0$ (say $y=0,0<x<25$ ), pile up at the vortex 
boundary and enter inside the solar wind plasma (the magnetospheric plasma) from many X-point regions connecting the islands, both on the left and on the right boundary of the vortex. This remains true even when the final vortex is somewhat disrupted at the end of the simulation, giving rise to a strip like mixed region in the interval $-25 \lesssim x \lesssim 25$ (right frame of Fig. 8). Indeed we observe many field lines crossing the two plasmas inside the central region starting from, or finally ending in, the full solar wind or magnetospheric side (see for example the field lines crossing at $x \approx-18, y \approx 37$, or $x \approx 15, y \approx 65$ ).

The role of the Hall term on the development of the reconnection instability has been outlined by performing a MHD run already introduced at the end of Sect. 3.1 (with $\eta=10^{-3}$ ). In this case the magnetic islands, shown in Fig. 3, right frame at $t=480$, do not form even if the same magnetic sheared layer between the vortices is dynamically generated by pairing. Indeed, in the MHD limit, the reconnection instability now driven by resistivity effects, proceeds at a too lower rate with respect to the dynamical time scale. As a consequence, reconnection is depressed by the dynamical modification of the large scale field lines advected by the flow, in agreement with the results obtained for an initially homogeneous in-plane magnetic field (Faganello et al., 2008c). On the other hand, the vortex scale magnetic islands generated by VIR at around $t \sim 300$ are observed also in the MHD limit. However, in this case the field connections between the solar wind and magnetospheric plasmas at the vortex borders are much less important thus reducing, in our opinion, the mixing efficiency and, again, being a signature of the decreasing of reconnection efficiency.

\section{Conclusions}

Solar wind interaction with the magnetosphere at low latitude magnetopause is an outstanding problem in space plasma physics, in particular concerning the understanding of the non-linear collisionless dynamics of a sheared flow in the presence of a magnetic field. Spacecraft observations become nowaday more and more accurate, but intrinsically limited by being a local source in a complex geometrical, multi-scale system. Therefore, numerical experiments must be considered as a basic complement for the understanding and interpretation of the satellite data. Numerical simulations, however, are limited by the impossibility of modelling the full physics in terms of frequencies and scale lengths involved in the system. Here, and in recent works (Faganello et al., 2008b,c), we adopted a two-fluids model that, far from being a realistic approach, gave us the possibility to study the onset of secondary reconnection instability induced by large scale MHD vortices starting with a homogeneous, or with a sheared, in-plane magnetic field. Until now, it has been generally assumed that the specific small scale process is "unimportant" for the global evolution provided reconnec- tion develops, the large scale evolution being then more or less unique. Here, instead, the most important result (see also Faganello et al., 2008c) is that there is a feedback of the small scale micro-physics allowing reconnection on the large scale evolution that, in turn, is responsible for the onset of such reconnection events. In other words, the global dynamics that sets the condition for reconnection to occur, can be significantly modified by the process at play at the microscale. Therefore, the study of kinetic effects that can compete at small scales is certainly today a very important research line in the field of magnetic reconnection. We must underline that the role of the reconnection microphysics capable of changing, or not, the large scale evolution has been proved in our study in a limited, but significant range of parameters, namely $25 \leq m_{i} / m_{e} \leq 100$ and $0.01 \leq \eta \leq 0.001$. Asymptotic values of the mass ratio and resistivity must be used to definitely prove our results. These simulations will be the object of future work. Finally, we have given evidence that even in those case where the Hall quadrupole is observed in the reconnection layer, it is very difficult to distinguish the guide field variations induced by the Hall effect from those produced by flow compression. One should therefore be careful when interpreting guide field fluctuations observed by satellite data as a clear signature of Hall reconnection.

Acknowledgements. We are pleased to acknowledge very interesting discussion with A. Mangeney, S. Landi and O. Le Contel. We acknowledge the super-computing center CINECA (Bologna), where part of the simulations was performed. This work is supported in part by PRIN-MIUR 2006.

Edited by: R. Pottelette

Reviewed by: two anonymous referees

\section{References}

Attico, N., Califano, F., and Pegoraro, F.: Fast collisionless reconnection in the whistler frequency range, Phys. Plasmas, 7, 23812387, doi:10.1063/1.874076, 2000.

Belmont, G. and Chanteur, G.: Kelvin-Helmholtz instability: nonlinear evolution, in: Turbulence and Nonlinear Dynamics in MHD Flows, edited by: Meneguzzi, M., Pouquet, A., and Sulem, P., Elsevier Science Publishers (North Holland), 1989.

Bian, N. and Vekstein, G.: On the two-fuid modification of the resistive tearing instability, Phys. Plasmas, 14, 120702, doi:10.1063/1.2820904, 2007.

Chen, L., Bhattacharjee, A., Puhl-Quinn, P. A., et al.: Observation of energetic electrons within magnetic islands, Nature Phys., 4, 19-23, doi:10.1038/nphys777, 2008.

Coppi, B.: Inertial instabilities in plasmas, Phys. Lett., 11, 226-228, doi:10.1016/0031-9163(64)90419-6, 1964.

Deng, X. H. and Matsumoto, H.: Rapid magnetic reconnection in the Earth's magnetosphere mediated by whistler waves, Nature, 410, 557-560, doi:10.1038/35069018, 2001.

Drake, J., Shay, M., and Swisdak, M.: The Hall fields and fast magnetic reconnection, Phys. Plasmas, 15, 042306, doi:10.1063/1.2901194, 2008 
Frey, H., Phan, T., Fuselier, S., and Mende, S.: Continuous magnetic reconnection at Earth's magnetopause, Nature, 426, 533, doi:10.1038/nature02084, 2003.

Faganello, M., Califano, F., and Pegoraro, F.: Competing Mechanisms of Plasma Transport in Inhomogeneous Configurations with Velocity Shear: The Solar-Wind Interaction with Earth's Magnetosphere, Phys. Rev. Lett., 100, 015001, doi:10.1103/PhysRevLett.100.015001, 2008.

Faganello, M., Califano, F., and Pegoraro, F.: Numerical Evidence of Undriven, Fast Reconnection in the Solar-Wind Interaction with Earth's Magnetosphere: Formation of Electromagnetic Coherent Structures, Phys. Rev. Lett., 101, 105001, doi:10.1103/PhysRevLett.101.105001, 2008.

Faganello, M., Califano, F., and Pegoraro, F.: Time window for magnetic reconnection in plasma configurations with velocity shear, Phys. Rev. Lett., 101, 175003, doi:10.1103/PhysRevLett.101.175003, 2008.

Furth, H. P., Killeen, J., Rosenbluth, M. N., et al.: FiniteResistivity Instabilities of a Sheet Pinch, Phys. Fluids, 6, 459484, doi:10.1063/1.1706761, 1963.

Hasegawa, H., Fujimoto, M., Phan, T.-D., et al.: Transport of solar wind into Earths magnetosphere through rolled-up KelvinHelmholtz vortices, Nature, 430, 755, doi:10.1038/nature02799, 2004.

Hasegawa, H., Sonnerup, B. U. Ö., Klecker, B., Paschmann, G., Dunlop, M. W., and Rème, H.: Optimal reconstruction of magnetopause structures from Cluster data, Ann. Geophys., 23, 973982, 2005,

http://www.ann-geophys.net/23/973/2005/.

Hesse, M. and Winske, D.: Hybrid Simulations of Collisionless Reconnection in Current Sheets, J. Geophys. Res., 99, 1117711192, doi:10.1029/94JA00676, 1998.

Landi, S., Velli, M., and Einaudi, G.: Alfvén Waves and Shock Wave Formation at an X-Point Magnetic Field Configuration, Astrophys. J., 624, 392-401, doi:10.1086/428822, 2005.

Liu, Z. X. and Hu, Y. D.: Local magnetic reconnection caused by vortices in the flow field, Geophys. Res. Lett., 15, 752-755, doi:10.1029/GL015i008p00752, 1988.

Mandt, M., Denton, R., and Drake, J.: Transition to Whistler Mediated Magnetic Reconnection, Geophys. Res. Lett., 21, 73-76, doi:10.1029/93GL03382, 1994.

Matsumoto, Y. and Hoshino, M.: Onset of turbulence induced by a Kelvin-Helmholtz vortex, Geophys. Res. Lett., 31, L02807, doi:10.1029/2003GL018195, 2004.

Miura, A.: Compressible magnetohydrodynamic Kelvin-Helmholtz instability with vortex pairing in the two-dimensional transverse configuration, Phys. Plasmas, 4, 2871-2885, doi:10.1063/1.872419, 1997.

Mozer, F. S., Bale, S. D., and Phan, T. D.: Evidence of Diffusion Regions at a Subsolar Magnetopause Crossing, Phys. Rev. Lett., 89, 015002, doi:10.1103/PhysRevLett.89.015002, 2004.
Nagai, T., Shinohara, I., and Fujimoto, M.: Geotail observations of the Hall current system: Evidence of magnetic reconnection in the magnetotail, J. Geophys. Res., 106, 25929-25950, doi:10.1029/2001JA900038, 2001.

Nykyri, K. and Otto, A.: Influence of the Hall term on KH instability and reconnection inside KH vortices, Ann. Geophys., 22, 935-949, 2004, http://www.ann-geophys.net/22/935/2004/.

Nykyri, K., Otto, A., Lavraud, B., Mouikis, C., Kistler, L. M., Balogh, A., and Rème, H.: Cluster observations of reconnection due to the Kelvin-Helmholtz instability at the dawnside magnetospheric flank, Ann. Geophys., 24, 2619-2643, 2006, http://www.ann-geophys.net/24/2619/2006/.

Nakamura, T. and Fujimoto, M.: Magnetic reconnection within MHD-scale Kelvin-Helmholtz vortices triggered by electron inertial effects, Adv. Space Res., 37, 522-526, doi:10.1016/j.asr.2005.01.057, 2006.

Oieroset, M., Phan, T. D., Fujimoto, M., et al: In situ detection of collisionless reconnection in the Earths magnetotail, Nature, 412, 414-417, doi:10.1038/35086520, 2001.

Otto, A. and Fairfield, D.: Kelvin-Helmholtz instability at the magnetotail boundary: MHD simulation and comparison with Geotail observations, J. Geophys. Res., 105, 21175-21190, doi:10.1029/1999JA000312, 2000.

Retinò, A., Sundkvist, D., Vaivads, A., et al.: In situ evidence of magnetic reconnection in turbulent plasma, Nature Phys., 3, 236238, doi:10.1038/nphys574, 2007.

Shay, M., Drake, J., Denton, R., and Biskamp, D.: Structure of the dissipation region during collisionless magnetic reconnection, J. Geophys. Res., 103, 9165-9176, doi:10.1029/97JA03528, 1998.

Shay, M., Drake, J., Rogers, B. N., Denton, R.: The Scaling of Collisionless, Magnetic Reconnection for Large Systems, Geophys. Res. Lett., 26, 2163-2166, doi:10.1029/1999GL900481, 1999.

Smyth, W. D.: Secondary Kelvin-Helmholtz instability in weakly stratified shear flow, J. Fluid Mech., 497, 67-98, 2003.

Uzdensky, D. A. and Kulsrud, R. M.: Physical origin of the quadrupole out-of-plane magnetic field in Hallmagnetohydrodynamic reconnection, Phys. Plasmas, 13, 062305, doi:10.1063/1.2210504, 2006.

Valentini, F., Trávncek, P., Califano, F., et al.: A Hybrid-Vlasov Model based on the Current Advance Method for the Simulation of Collisionless Magnetized Plasma, J. Comp. Phys., 225, 753770, doi:10.1016/j.jcp.2007.01.001, 2007.

Vaivads, A., Khotyaintsev, Y., André, M., et al.: Structure of the Magnetic Reconnection Diffusion Region from Four-Spacecraft Observations, Phys. Rev. Lett., 93, 105001, doi:10.1103/PhysRevLett.93.105001, 2004.

Vekstein, G. and Bian, N.: Hall assisted forced magnetic reconnection, Phys. Plasmas, 13, 122105, doi:10.1063/1.2398933, 2000. 